OPEN ACCESS

Edited by:

Anupam Mitra,

UC Davis Health, United States

Reviewed by:

Marina Venturini,

Civil Hospital of Brescia, Italy

Jose-manuel Carrascosa Hospital Germans Trias i Pujol, Spain

${ }^{*}$ Correspondence:

Sandeep Arora

aroraderma@gmail.com

Specialty section:

This article was submitted to

Dermatology,

a section of the journal

Frontiers in Medicine

Received: 17 April 2021

Accepted: 28 July 2021

Published: 19 August 2021

Citation:

Arora S, Das P and Arora G (2021)

Systematic Review and

Recommendations to Combine Newer

Therapies With Conventional Therapy in Psoriatic Disease.

Front. Med. 8:696597.

doi: 10.3389/fmed.2021.696597

\section{Systematic Review and Recommendations to Combine Newer Therapies With Conventional Therapy in Psoriatic Disease}

\author{
Sandeep Arora ${ }^{*}$, Pankaj Das ${ }^{1}$ and Gulhima Arora ${ }^{2}$ \\ ${ }^{1}$ Department of Dermatology, Army College of Medical Sciences \& Base Hospital Delhi Cantt, New Delhi, India, ${ }^{2}$ Consultant \\ Dermatologist, Mehektagul Dermaclinic, New Delhi, India
}

Background: Psoriasis continues to have unmet needs in its management despite introduction of newer molecules. Monotherapy with these newer agents may not achieve therapeutic goals in all cases, hence necessitating their combinations with other molecules. Improved understanding of newer as well as conventional treatment modalities and experiences in their combinations hence necessitates therapeutic guidelines for their use in psoriasis.

Objective: To review the combinations of treatments reported in literature and recommendations for their use based on best current evidence in literature.

Methods: A literature review of MEDLINE database for studies evaluating combinations of newer therapies with conventional therapies in psoriasis was done. Newer therapies were identified as biologic disease modifying anti rheumatic drugs and other molecules such as apremilast while conventional therapies included methotrexate, cyclosporine, or retinoids, phototherapy and others. The therapeutic guidelines are proposed with the aim to provide evidenced based approach to combine newer and conventional agents in day-to-day psoriasis management.

Findings: Combination of acitretin and narrow band ultraviolet B (NB-UVB)/Psoralen with ultraviolet $A$ (PUVA) achieves faster clearance and allows reduction of dose of the latter. A variable outcome is reported of methotrexate with TNF- $\alpha$ inhibitors vs. TNF- $\alpha$ inhibitors alone, although addition of methotrexate appears to reduce immunogenicity of TNF- $\alpha$ inhibitors thereby preventing formation of anti-drug antibodies especially in case of infliximab. While combination of acitretin and PUVA is beneficial, combining TNF- $\alpha$ inhibitors and phototherapy too produces better and faster results but long term risks of Non Melanoma Skin Cancers (NMSCs) may preclude their use together. Combination of cyclosporine and phototherapy is not recommended due to greater chances of NMSCs. Adding phototherapy to Fumaric Acid Esters (FAEs) improves efficacy. Apremilast can be safely combined with available biologic agents in patients with plaque psoriasis or psoriatic arthritis not responding adequately to biologics alone. Hydroxyurea and acitretin may be used together increasing their efficacy and reducing doses of both and hence their adverse effects. 
Conclusion: Selected clinical scenarios shall benefit from combinations therapies, improving efficacy of both conventional and newer agents and the same time helping reduce toxicity of higher dosages when used individually.

Keywords: psoriasis, combination (combined) therapy, conventional therapy, biologics, guidelines and recommendations

\section{INTRODUCTION}

Psoriasis is a chronic relapsing-remitting inflammatory papulosquamous disease, which affects $\sim 0.51-11.43 \%$ of adults worldwide (1). This immune-mediated disease causes chronic inflammation in milieu which not only affects skin, but also joints, blood vessels, heart, liver, and kidneys (2) as well as metabolic syndrome $(3,4)$. PsA (Psoriatic Arthritis) may be present in $>40 \%$ of psoriasis patients leading to joint damage and deformities thereby severely affecting QoL (Quality of Life) and physical functioning (5-7). Early diagnosis and treatment intervention are crucial for optimal patient care $(8,9)$. The chronic relapsing course of disease with these co-morbidities are associated with increased physical and psychological burden, which leads to impaired Quality of Life (QoL) and depression (10). Mild psoriasis responds to topical therapy while moderate to severe psoriasis may need augmentation with phototherapy or systemic agents. Severe psoriasis may sometimes be refractory to one systemic agents requiring combination with another to maintain remission $(11,12)$. Combining therapeutic agents holds potential in synergistic action for a better control over disease activity. Moreover, a combination may be needed to reduce adverse effects by allowing reduction of dose despite severe disease. However, combining therapies pose challenges in tolerability, acute and long-term adverse effects in the absence of clear overall guidelines. Conventionally, immunosuppressive and non-biologic disease modifying immune-modulatory drugs such as methotrexate, cyclosporine, retinoids, phototherapy, and others have been used. Management of psoriasis has been revolutionized by biologics which have improved management of psoriasis but aren't panaceas either. Combining newer and conventional therapies provide a tantalizing option for managing psoriasis, to achieve prolonged remission and better Quality of Life (QoL). Although there are numerous Randomized Control Trials (RCTs), case series, case reports, and expert opinions proving efficacy of different combinations in various clinical scenarios, literature is lacking in clear cut guidelines on how and when to combine the newer and conventional therapeutic options. This review aims at analyzing data available from studies with highest quality of evidence i.e., RCTs and generate recommendations for combining newer and conventional therapies in psoriatic disease.

\section{MATERIALS AND METHODS}

\section{Protocol Development and Eligibility Criteria}

A protocol was designed and followed as laid down by PRISMA (Preferred Reporting Items for Systematic Review and Meta-Analyses) statement (Figure 1). The conventional therapies considered being immunosuppressive and nonbiologic disease modifying immune-modulatory drugs such as methotrexate, cyclosporine, retinoids, phototherapy, hydroxyurea, and fumaric acid esters (13). The newer therapies were identified as biologic disease modifying antirheumatic drugs namely- TNF $\alpha$ (Tumor Necrosis Factor- $\alpha$ ) inhibitors- etanercept, adalimumab, infliximab, golimumab, and certolizumab pegol; IL-17A (Interleukin-17A) inhibitorssecukinumab and ixekizumab; IL-17RA (Interleukin-17 Receptor Antagonist)- brodalumab; IL-12/IL-23 inhibitor- ustekinumab, IL-23 inhibitor- guselkumab; oral PDE-4 (Phosphodiesterase-4) inhibitor- apremilast and tofacitinib selective JAK (Janus Kinase) 1 and 3 inhibitor (14).

\section{Search Strategy}

A literature search was performed for studies conducted in psoriasis therapeutics published before 01 Jan 2021. MEDLINE (OVID, from 1948), EMBASE (OVID, from 1980), Cochrane Central Register of Controlled Trials (CENTRAL), ahead of print subset fraction from Pubmed- not yet published in (OVID MEDLINE), and ongoing trial registries (http://clinicaltrials. gov/) were searched with no language restrictions. The search was carried out through use of keywords targeting all drugs used in conventional as well as newer therapies. In MEDLINE and EMBASE, a methodologic filter for search was used to identify RCTs and clinical controlled trials in Medical Subject Headings and titles and abstracts (adapted from the Cochrane Central Register of Controlled Trials). For potential drug combinations where RCTs were not found, the search was extended to include lower tiers of hierarchy of evidence up to case series. A systematic method in search was used for each database to broaden the search through inclusion of pertinent search terms as relevant citations were recognized (i.e., by scrutinizing references and citing articles).

\section{Search Terms}

The search terms which were used are as follows: ("methotrexate" OR "cyclosporine" OR "ciclosporine" OR "acitretin" OR "phototherapy" OR "hydroxyurea" OR "fumaric acid esters" OR “conventional" [MeSH term] OR "drugs" [MeSH term] OR "etanercept" OR "infliximab" OR "adalimumab" OR "secukinumab" OR "golimumab" OR “ixekizumab” OR "ustekinumab" OR "guselkumab" OR “certolizumab pegol” OR “apremilast” OR “tofacitinib” OR "biologics” [MeSH term] OR “psoriasis” [MeSH term] OR “combination” [MeSH term] OR "therapy" [MeSH term]). 

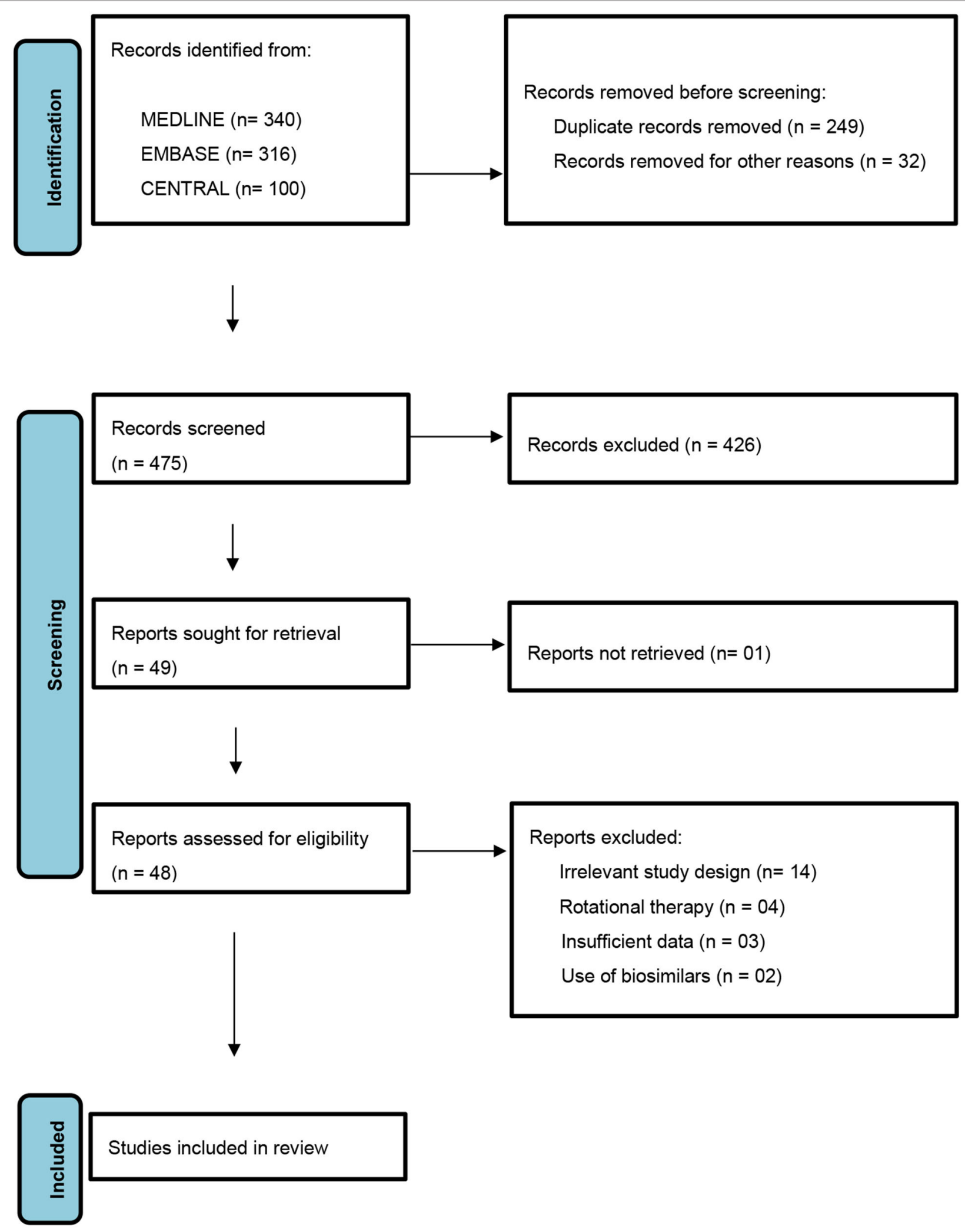

FIGURE 1 | PRISMA flow diagram for selection of studies. 


\section{Inclusion Criteria}

Randomized Controlled Trials $(N>10)$ which reported on the efficacy and safety of combined use of conventional and newer drugs in psoriatic disease were included. Potential combinations in which RCTs have not been carried out, studies with lower levels of evidence were also included.

\section{Exclusion Criteria}

In-vitro, preclinical and animal studies, case reports and expert opinions were excluded from the review as well as all studies not meeting the inclusion criteria. Studies with rotational or sequential therapies using these drugs as well as those combining alternative medicines (i.e., Chinese herbal) were excluded. Biosimilars were excluded from the study to maintain uniformity on drug efficacy data.

\section{Selection of Studies}

Using the above keywords, the titles and abstracts from electronic literature search were screened, and full text of articles that met the pre-defined inclusion criteria were obtained. Successively, articles were scanned for inclusion or exclusion. The selection of studies were implemented by 2 reviewers independent of each other (S.A. and P.D.). The quality of each included articles was assessed in agreement with the Cochrane handbook of systematic reviews of interventions 5.1.0 (updated March 2011). Any disagreements between the two reviewers were resolved by commonly drawn consensus by discussion or intervention by a third reviewer (G.A.).

\section{Data Extraction}

Information on the year of publication, study design, study reference, number of patients $(\mathrm{N})$, baseline disease severity, treatment schedule, duration of combination therapy, and period of follow-up were extracted. Critical as well as important outcomes were carefully chosen to determine the quality of evidence. Critical outcomes were defined as the proportion of patients who attained a PASI (Psoriasis Area and Severity Index) of 90, PASI of 75, and a PGA (Physician Global Assessment) of clear or almost clear; discontinuation of a particular drug because of AEs (Adverse Effects); proportion of patients who encountered SAEs (Serious Adverse Events); and mean change in DLQI (Dermatology Life Quality Index). Important outcomes were defined as lack of efficacy leading to withdrawals (number), proportion of patients with AEs (not leading to drug withdrawal), mean time to clearance, mean change in PASI $(0-72,0-18$, and 0-16) and mean time to relapse.

\section{RESULTS}

Our literature search yielded 25 RCTs (Table 1) combining different drugs which met the criteria to be included for analysis in the present study. Potential drug combinations for which RCTs have not been done, studies with lesser levels of evidence up to case series were searched for to look for evidence and gaps in research (Table 2).

\section{Discussion and Recommendations}

Literature search yielded 25 RCTs combining different agents to treat psoriasis and/or psoriatic arthropathy. Most of the studies involved combinations with Narrow Band Ultraviolet B (NBUVB) /Psoralen with Ultraviolet A (PUVA) or methotrexate.

\section{NB-UVB/PUVA and Acitretin}

There are 02 RCTs involving acitretin and UVB/PUVA by Tanew et al. (15) and Lowe et al. (16). The former found that the cumulative PUVA dose required for complete clearance in PUVA-acitretin group was $58.7 \pm 17.9 \mathrm{~J} / \mathrm{cm}^{2}$ whereas in PUVAplacebo group was $101.5 \pm 15.8 \mathrm{~J} / \mathrm{cm}^{2}$. In RCT by Lowe et al. (16), 14 participants in the UVB-acitretin group took a total of 873 min of UVB exposure for complete clearance as compared to a significantly higher time- 1,236 min in the UVB-placebo group $(n=15)$. At the end of 12 weeks, the mean PASI \pm SD in acitretin + UVB group reduced significantly from $8.83 \pm 1.8$ to $2.27 \pm$ 1.04 ( $\mathrm{p}<0.01$ ), whereas it reduced from $9.75 \pm 2.34$ to $6.36 \pm$ 3.07 in placebo + UVB group. Both the RCTs concluded that adding UVB/PUVA to acitretin achieves greater as well as faster clearance than either placebo- UVB/PUVA or acitretin alone. Clinical adverse effects of added acitretin in both the studies were generally well-tolerated and similar to previous studies in treatment of psoriasis with acitretin (46-48).

\section{Recommendation}

We recommend combining these two modalities when patients do not respond to either one of the two. In addition to increased efficacy, the combination allows reduction of cumulative dose of $\mathrm{UVB} / \mathrm{PUVA}$. Also important is the prevention of non-melanoma skin cancers by acitretin which may be caused by long term UVB/PUVA $(49,50)$.

\section{NB-UVB With TNF $\alpha$ Inhibitors}

Etanercept with NB-UVB combination has been evaluated by Lynde et al. (17), Park et al. (18), Calzavara-Pinton et al. (19), and Gambichler et al. (20). Lynde et al. (17) concluded that addition of NB-UVB to etanercept did not significantly improve the overall clinical response except for a subset of patients with high adherence to NB-UVB without increasing the adverse effects significantly. Park et al. (18) studied combination of etanercept and NB-UVB in obese patients. They concluded that the combination has a similar efficacy to etanercept monotherapy even in the setting of obesity. However, Calzavara-Pinton et al. (19) who performed an intra-individual RCT in receiving etanercept and a randomized half of the body with NB-UVB for found that The PSI (Psoriasis Severity Index) scores of nonirradiated control lesions were $6.4 \pm 2.3$ and $5.8 \pm 2.5$ ( $p=$ not significant) before and after the treatment respectively, whereas the PSI of irradiated psoriatic plaques were $6.3 \pm 2.3$ and 0.5 $\pm 0.8(p<0.05)$. In the combination group, the mean PASI \pm $\mathrm{SD}$ value reduced from $16.2 \pm 9.2$ to $2.4 \pm 2.8$ in 12 weeks. The patients received $14.6 \pm 3.3$ exposures resulting in a cumulative dose of $8.4 \pm 4.2 \mathrm{~J} \mathrm{~cm}^{-2}$. While the combined treatment was always well-tolerated, it was aimed at short duration of NBUVB therapy for faster clearance to avoid long term adverse effects. It also may help reduce total doses as well as cost of 
TABLE 1 | Randomized controlled trials which met the inclusion criteria and selected in this study $(n=25)$.

\begin{tabular}{|c|c|c|c|c|c|c|c|c|c|c|c|}
\hline \multirow[t]{2}{*}{ S no. } & \multirow[t]{2}{*}{ References } & \multirow[t]{2}{*}{ Study design } & \multirow{2}{*}{$\begin{array}{c}\text { No of } \\
\text { patients }\end{array}$} & \multirow{2}{*}{$\begin{array}{l}\text { Baseline disease } \\
\text { severity }\end{array}$} & \multirow[t]{2}{*}{ Intervention } & \multirow[t]{2}{*}{ Control group(s) } & \multirow{2}{*}{$\begin{array}{l}\text { Study length } \\
\text { (weeks) }\end{array}$} & \multirow{2}{*}{$\begin{array}{l}\text { Follow up } \\
\text { (weeks) }\end{array}$} & \multicolumn{2}{|c|}{ Outcome measures used in study analysis } & \multirow[t]{2}{*}{ LoE } \\
\hline & & & & & & & & & Efficacy & Safety & \\
\hline 1. & Tanew et al. (15) & $\begin{array}{l}\text { Randomized } \\
\text { double blinded } \\
\text { trial }\end{array}$ & 60 & $\geq 20 \% \mathrm{BSA}$ or $\mathrm{PASI} \geq 10$ & $\begin{array}{l}\text { Acitretin } 1 \mathrm{mg} \text { per } \mathrm{kg} \\
\text { per day plus four } \\
\text { PUVA exposures per } \\
\text { week }\end{array}$ & $\begin{array}{l}\text { Placebo plus four } \\
\text { PUVA exposures per } \\
\text { week }\end{array}$ & $\begin{array}{l}\text { Until complete } \\
\text { clearance/maximum } \\
\text { of } 11 \text { weeks. }\end{array}$ & 11 & $\begin{array}{l}\text { Complete remission or marked } \\
\text { improvement i.e.at least } 90 \% \\
\text { clearing of psoriasis }\end{array}$ & $\begin{array}{l}\text { Percentage of patients } \\
\text { with AEs and } \\
\text { SAES/ withdrawal } \\
\text { because of AEs }\end{array}$ & $2 \mathrm{~b}$ \\
\hline 2. & Lowe et al. (16) & $\begin{array}{l}\text { Randomized } \\
\text { controlled trial }\end{array}$ & 37 & $\begin{array}{l}\text { Moderate to severe chronic } \\
\text { plaque type psoriasis }\end{array}$ & $\begin{array}{l}\text { Acitretin } 50 \mathrm{mg} \text { per } \\
\text { day plus UVB }\end{array}$ & Placebo plus UVB & 12 & 12 & $\begin{array}{l}\text { Mean PASI at the end of } 12 \\
\text { weeks }\end{array}$ & $\begin{array}{l}\text { Percentage of patients } \\
\text { with AEs }\end{array}$ & $2 \mathrm{~b}$ \\
\hline 3. & Lynde et al. (17) & $\begin{array}{l}\text { Single- blinded } \\
\text { randomized } \\
\text { controlled trial }\end{array}$ & 99 & $\geq 10 \% \mathrm{BSA}$ or $\mathrm{PASI} \geq 10$ & $\begin{array}{l}\text { Etanercept } 50 \mathrm{mg} \\
\text { once a week plus } \\
\text { thrice weekly NB-UVB }\end{array}$ & $\begin{array}{l}\text { Etanercept } 50 \mathrm{mg} \\
\text { once a week }\end{array}$ & 24 & 24 & $\begin{array}{l}\text { PASI 90, PASI } 75 \text {, PGA- clear, } \\
\text { minimal, mild, moderate, severe, } \\
\text { very severe BSA and DLQI }\end{array}$ & $\begin{array}{l}\text { AEs, SAEs infectious } \\
\text { adverse events and } \\
\text { injection-site reactions }\end{array}$ & $1 \mathrm{~b}$ \\
\hline 4. & Park et al. (18) & $\begin{array}{l}\text { Randomized, } \\
\text { 'head-to-head' } \\
\text { pilot trial }\end{array}$ & 30 & $\begin{array}{l}\geq 10 \% \mathrm{BSA} \text { or PASI } \geq 10 \\
\text { with BMI of } 30 \text { or greater }\end{array}$ & $\begin{array}{l}\text { Etanercept induction } \\
\text { dose at } 50 \mathrm{mg} \text { twice } \\
\text { weekly for } 12 \text { weeks } \\
\text { followed by } \\
\text { combination of } \\
\text { etanercept at } \\
\text { maintenance dose of } \\
50 \text { mg weekly with } \\
\text { NB-UVB thrice weekly }\end{array}$ & $\begin{array}{l}\text { Etanercept induction } \\
\text { dose at } 50 \mathrm{mg} \text { twice } \\
\text { weekly for } 12 \text { weeks } \\
\text { followed by } \\
\text { etanercept } \\
\text { monotherapy at } \\
\text { maintenance dose of } \\
50 \text { mg weekly }\end{array}$ & 24 & 24 & $\begin{array}{l}\text { (i) PASI } 75 \text { response after } 12 \\
\text { weeks of combination } \\
\text { etanercept and NB-UVB therapy } \\
\text { (ii)Improvement in average PASI, } \\
\text { (iii)Improvement in BSA and (iv) } \\
\text { Improvement in PGA }\end{array}$ & $\begin{array}{l}\text { Serious Adverse Events } \\
\text { (SAEs) at weeks } 12 \text { and } \\
24 .\end{array}$ & $1 \mathrm{~b}$ \\
\hline 5. & $\begin{array}{l}\text { Calzavara- } \\
\text { Pinton et al. } \\
\text { (19) }\end{array}$ & $\begin{array}{l}\text { Randomized } \\
\text { controlled } \\
\text { intra-individual } \\
\text { trial }\end{array}$ & 20 & $\begin{array}{l}\text { PASI } \geq 10 \text {, Patients on } \\
\text { etanercept alone who did } \\
\text { not achieve PASI } 75 \text { within } \\
12 \text { weeks }\end{array}$ & $\begin{array}{l}\text { Etanercept at } 50 \mathrm{mg} \\
\text { twice weekly plus } \\
\text { NB-UVB thrice } \\
\text { weekly on a selected } \\
\text { psoriatic plaque }\end{array}$ & $\begin{array}{l}\text { Covered plaque } \\
\text { served as } \\
\text { non-irradiated control }\end{array}$ & 24 & 24 & $\begin{array}{l}\text { Mean PASI reduction, PASI 90, } \\
\text { PASI } 75\end{array}$ & $\begin{array}{l}\text { Percentage of patients } \\
\text { with AEs }\end{array}$ & $2 \mathrm{~b}$ \\
\hline 6. & $\begin{array}{l}\text { Gambichler } \\
\text { et al. (20) }\end{array}$ & $\begin{array}{l}\text { Randomized } \\
\text { controlled } \\
\text { intra-individual } \\
\text { trial }\end{array}$ & 14 & $\mathrm{PASI} \geq 10$ & $\begin{array}{l}\text { Etanercept at } 50 \mathrm{mg} \\
\text { twice weekly plus } \\
\text { NB-UVB thrice } \\
\text { weekly on a selected } \\
\text { psoriatic plaque }\end{array}$ & $\begin{array}{l}\text { Covered plaque } \\
\text { served as } \\
\text { non-irradiated control }\end{array}$ & 6 & 6 & $\begin{array}{l}\text { Modified PASI reduction, } \\
\text { performance of skin biopsies }\end{array}$ & $\begin{array}{l}\text { Percentage of patients } \\
\text { with AEs }\end{array}$ & $2 \mathrm{~b}$ \\
\hline 7. & Wolf et al. (21) & $\begin{array}{l}\text { Open-label } \\
\text { randomized trial }\end{array}$ & 10 & $\mathrm{PASI} \geq 10$ & $\begin{array}{l}\text { Ustekinumab at } 45 \text { or } \\
90 \mathrm{mg} \text { at week } 0 \text { and } \\
4 \text { and plus NB- thrice } \\
\text { weekly }\end{array}$ & $\begin{array}{l}\text { Ustekinumab at } 45 \text { or } \\
90 \mathrm{mg} \text { at week } 0 \text { and } \\
4\end{array}$ & 6 & 12 & PASI of 75 , mean change in PASI & $\begin{array}{l}\text { Percentage of patients } \\
\text { with AEs, withdrawal } \\
\text { because of AEs }\end{array}$ & $2 \mathrm{~b}$ \\
\hline 8. & $\begin{array}{l}\text { Mahajan et al. } \\
\text { (22) }\end{array}$ & $\begin{array}{l}\text { Randomized, } \\
\text { single blinded, } \\
\text { placebo } \\
\text { controlled trial }\end{array}$ & 40 & $\geq 10 \% \mathrm{BSA}$ & $\begin{array}{l}\text { Methotrexate at } \\
0.5 \mathrm{mg} \text { per } \mathrm{kg} \text { once } \\
\text { weekly to a maximum } \\
\text { of } 30 \mathrm{mg} \text { per week } \\
\text { plus NB-UVB thrice } \\
\text { weekly }\end{array}$ & $\begin{array}{l}\text { Placebo plus NB-UVB } \\
\text { thrice weekly }\end{array}$ & 24 & 24 & PASI 75, PASI 50 & $\begin{array}{l}\text { Percentage of patients } \\
\text { with AEs and } \\
\text { SAEs/withdrawal } \\
\text { because of AEs. }\end{array}$ & $2 \mathrm{~b}$ \\
\hline 9. & $\begin{array}{l}\text { Asawanonda } \\
\text { et al. (23) }\end{array}$ & $\begin{array}{l}\text { Open-label } \\
\text { randomized trial }\end{array}$ & 24 & $\geq 20 \% \mathrm{BSA}$ & $\begin{array}{l}\text { Methotrexate at } \\
15 \mathrm{mg} \text { per week plus } \\
\text { NB-UVB thrice weekly }\end{array}$ & $\begin{array}{l}\text { Placebo plus NB-UVB } \\
\text { thrice weekly }\end{array}$ & 24 & 24 & $\begin{array}{l}\text { PASI 90, PASI 50, Dermatology } \\
\text { Life Quality Index (DLQI) }\end{array}$ & $\begin{array}{l}\text { Percentage of patients } \\
\text { with AEs and SAES }\end{array}$ & $1 \mathrm{~b}$ \\
\hline 10. & $\begin{array}{l}\text { Al-Hamamy } \\
\text { et al. (24) }\end{array}$ & $\begin{array}{l}\text { Open-label } \\
\text { randomized trial }\end{array}$ & 120 & $\geq 10 \% \mathrm{BSA}$ & $\begin{array}{l}\text { Methotrexate at } \\
0.2 \mathrm{mg} \text { per } \mathrm{kg} \text { weekly } \\
\text { with a maximum of } \\
20 \mathrm{mg} \text { per week plus } \\
\text { NB-UVB thrice weekly }\end{array}$ & $\begin{array}{l}\text { (i) Methotrexate at } \\
0.2 \mathrm{mg} \text { per } \mathrm{kg} \text { weekly } \\
\text { with a maximum of } \\
20 \mathrm{mg} \text { per week (ii) } \\
\text { NB-UVB thrice weekly }\end{array}$ & 24 & 48 & PASI 90, PASI 50 & $\begin{array}{l}\text { Percentage of patients } \\
\text { with AEs and SAEs }\end{array}$ & $2 \mathrm{~b}$ \\
\hline 11. & $\begin{array}{l}\text { Zachariae et al. } \\
\text { (25) }\end{array}$ & $\begin{array}{l}\text { Open-label } \\
\text { randomized trial }\end{array}$ & 60 & $\geq 10 \%$ BSA or $\mathrm{PASI} \geq 8$ & $\begin{array}{l}\text { Etanercept } 50 \mathrm{mg} \\
\text { twice weekly for } 12 \\
\text { weeks, and then } \\
25 \mathrm{mg} \text { twice weekly } \\
\text { for } 12 \text { weeks plus } \\
\text { continued } \\
\text { methotrexate therapy }\end{array}$ & $\begin{array}{l}\text { Etanercept } 50 \mathrm{mg} \\
\text { twice weekly for } 12 \\
\text { weeks, and then } \\
25 \mathrm{mg} \text { twice weekly } \\
\text { for } 12 \text { weeks plus } \\
\text { methotrexate tapered } \\
\text { and discontinued } \\
\text { during the } 4 \text { weeks }\end{array}$ & 24 & 24 & $\begin{array}{l}\text { Physician's Global Assessment } \\
\text { (PGA), PASI 50, PASI 75, PASI } \\
\text { 90, DLQI. }\end{array}$ & $\begin{array}{l}\text { Percentage of patients } \\
\text { with AEs and } \\
\text { SAEs/withdrawal } \\
\text { because of AEs }\end{array}$ & $2 \mathrm{~b}$ \\
\hline
\end{tabular}


TABLE 1 | Continued

\begin{tabular}{|c|c|c|c|c|c|c|c|c|c|c|c|}
\hline \multirow[t]{2}{*}{ S no. } & \multirow[t]{2}{*}{ References } & \multirow[t]{2}{*}{ Study design } & \multirow{2}{*}{$\begin{array}{c}\text { No of } \\
\text { patients }\end{array}$} & \multirow{2}{*}{$\begin{array}{l}\text { Baseline disease } \\
\text { severity }\end{array}$} & \multirow[t]{2}{*}{ Intervention } & \multirow[t]{2}{*}{ Control group(s) } & \multirow{2}{*}{$\begin{array}{l}\text { Study length } \\
\text { (weeks) }\end{array}$} & \multirow{2}{*}{$\begin{array}{l}\text { Follow up } \\
\text { (weeks) }\end{array}$} & \multicolumn{2}{|c|}{ Outcome measures used in study analysis } & \multirow[t]{2}{*}{ LoE } \\
\hline & & & & & & & & & Efficacy & Safety & \\
\hline 12. & $\begin{array}{l}\text { Gottlieb et al. } \\
\text { (26) }\end{array}$ & $\begin{array}{l}\text { Randomized, } \\
\text { double-blind, } \\
\text { placebo } \\
\text { controlled trial }\end{array}$ & 478 & $\geq 10 \%$ BSA or PASI $\geq 10$ & $\begin{array}{l}\text { Etanercept } 50 \mathrm{mg} \\
\text { subcutaneously twice } \\
\text { weekly for } 12 \text { weeks } \\
\text { followed by } 50 \mathrm{mg} \\
\text { once weekly for } 12 \\
\text { weeks plus } \\
\text { methotrexate titrated } \\
\text { from } 7.5 \mathrm{mg} \text { to } \\
\text { maximum of } 15 \mathrm{mg} \text { or } \\
\text { tolerated dose. }\end{array}$ & $\begin{array}{l}\text { Etanercept } 50 \mathrm{mg} \\
\text { subcutaneously twice } \\
\text { weekly for } 12 \text { weeks } \\
\text { followed by } 50 \mathrm{mg} \\
\text { once weekly for } 12 \\
\text { weeks plus placebo }\end{array}$ & 24 & 24 & $\begin{array}{l}\text { PASI 90, PASI 75, PASI 50, static } \\
\text { Physician's Global Assessment } \\
\text { (SPGA), BSA improvement from } \\
\text { baseline at weeks } 12 \text { and } 24 . \\
\text { Assessments were performed at } \\
\text { screening, at baseline, and every } \\
4 \text { weeks thereafter throughout } \\
\text { the study. }\end{array}$ & $\begin{array}{l}\text { Percentage of patients } \\
\text { with AEs. }\end{array}$ & $1 \mathrm{~b}$ \\
\hline 13. & Yu et al. (27) & $\begin{array}{l}\text { Randomized } \\
\text { trial, unclear } \\
\text { blinding }\end{array}$ & 30 & $\mathrm{PASI} \geq 10$ & $\begin{array}{l}\text { Etanercept } 50 \mathrm{mg} \\
\text { once weekly plus oral } \\
\text { methotrexate } \\
7.5-15 \mathrm{mg} \text { per week }\end{array}$ & $\begin{array}{l}\text { Etanercept } 50 \mathrm{mg} \\
\text { once weekly }\end{array}$ & 24 & 24 & $\begin{array}{l}\text { PASI score, static Physician's } \\
\text { Global Assessment (sPGA), } \\
\text { Patient's Global Assessment } \\
\text { (PtGA), Dermatology Life Quality } \\
\text { Index (DLQl) }\end{array}$ & $\begin{array}{l}\text { Percentage of patients } \\
\text { with AEs. }\end{array}$ & $2 \mathrm{~b}$ \\
\hline 14. & Mease et al. (28) & $\begin{array}{l}\text { Randomized, } \\
\text { double-blind, } \\
\text { placebo- } \\
\text { controlled triple } \\
\text { armed trial }\end{array}$ & 851 & $\begin{array}{l}3 \text { tender joints and } 3 \\
\text { swollen joints (based on } \\
68 \text { - and } 66 \text {-joint, and an } \\
\text { active psoriatic skin lesion } \\
\text { that was } \geq 2 \mathrm{~cm} \text { in } \\
\text { diameter). }\end{array}$ & $\begin{array}{l}\text { Etanercept (target } \\
\text { dose } 50 \mathrm{mg} \text { ) plus oral } \\
\text { methotrexate (target } \\
\text { dose } 20 \mathrm{mg} \text { ) given } \\
\text { weekly. }\end{array}$ & $\begin{array}{l}\text { Methotrexate (target } \\
\text { dose } 20 \mathrm{mg} \text { ) plus } \\
\text { subcutaneous } \\
\text { placebo given weekly } \\
\text { or subcutaneous } \\
\text { etanercept (target } \\
\text { dose } 50 \mathrm{mg} \text { ) plus oral } \\
\text { placebo given weekly. }\end{array}$ & 48 & 48 & $\begin{array}{l}\text { ACR20, Minimal Disease Activity } \\
\text { (MDA) response, Leeds Dactylitis } \\
\text { Index (LDI), static Physician's } \\
\text { Global Assessment (sPGA). }\end{array}$ & $\begin{array}{l}\text { Percentage of patients } \\
\text { with AEs and } \\
\text { SAEs/withdrawal } \\
\text { because of AEs. }\end{array}$ & $1 \mathrm{~b}$ \\
\hline 15. & $\begin{array}{l}\text { Baranauskaite } \\
\text { et al. (29) }\end{array}$ & $\begin{array}{l}\text { Open-label } \\
\text { randomized trial }\end{array}$ & 115 & $\begin{array}{l}\text { Psoriasis and psoriatic } \\
\text { arthropathy }\end{array}$ & $\begin{array}{l}\text { Inffiximab } 5 \mathrm{mg} \text { per } \mathrm{kg} \\
\text { infusions at weeks } 0 \text {, } \\
2,6, \text { and } 14 \text { plus } \\
\text { methotrexate } 15 \mathrm{mg} \\
\text { per week }\end{array}$ & $\begin{array}{l}\text { Methotrexate } 15 \mathrm{mg} \\
\text { per week }\end{array}$ & 16 & 16 & $\begin{array}{l}\text { ACR20, ACR50 and ACR70 } \\
\text { responses, PASI 75, PASI 90, } \\
\text { EULAR response, physician and } \\
\text { patient global assessment of } \\
\text { disease activity, disease activity } \\
\text { score in } 28 \text { joints (DAS28) } \\
\text { scores, minimal disease activity } \\
\text { (MDA) }\end{array}$ & $\begin{array}{l}\text { Percentage of patients } \\
\text { with AEs and } \\
\text { SAEs/withdrawal } \\
\text { because of AEs. }\end{array}$ & $2 \mathrm{~b}$ \\
\hline 16. & $\begin{array}{l}\text { van Mens et al. } \\
(30)\end{array}$ & $\begin{array}{l}\text { Randomized, } \\
\text { double-blind, } \\
\text { placebo } \\
\text { controlled trial }\end{array}$ & 59 & $\begin{array}{l}\text { Patients meeting CASPAR } \\
\text { criteria and current active } \\
\text { disease, defined as the } \\
\text { presence of at least three } \\
\text { swollen and three tender } \\
\text { joints. }\end{array}$ & $\begin{array}{l}\text { Methotrexate } 25 \mathrm{mg} \\
\text { per week or as } \\
\text { tolerated plus } \\
\text { Golimumab } 50 \mathrm{mg} \\
\text { administered every } 4 \\
\text { weeks }\end{array}$ & $\begin{array}{l}\text { Methotrexate } 25 \mathrm{mg} \\
\text { per week or as } \\
\text { tolerated plus } \\
\text { placebo prefilled } \\
\text { syringes } \\
\text { administrated every } 4 \\
\text { weeks }\end{array}$ & 22 & 22 & $\begin{array}{l}\text { Disease Activity Score (DAS), } \\
\text { MDA, ACR2O/50/70 responses, } \\
\text { Leeds Enthesitis Index, and } \\
\text { Dermatology Life Quality Index } \\
\text { (DLQI). }\end{array}$ & $\begin{array}{l}\text { Percentage of patients } \\
\text { with AEs and } \\
\text { SAEs/withdrawal } \\
\text { because of AEs. }\end{array}$ & $2 \mathrm{~b}$ \\
\hline 17. & $\begin{array}{l}\text { Vieira-Sousa } \\
\text { et al. (31) }\end{array}$ & $\begin{array}{l}\text { Randomized, } \\
\text { double-blind, } \\
\text { placebo } \\
\text { controlled trial }\end{array}$ & 48 & $\begin{array}{l}\text { Classification for Psoriatic } \\
\text { Arthritis criteria } \geq 1 \text { digit } \\
\text { with tender dactylitis and } \\
\geq 1 \text { other site of active } \\
\text { inflammation (joints, } \\
\text { enthesis, spine, skin, or } \\
\text { nails). }\end{array}$ & $\begin{array}{l}\text { Methotrexate } 25 \mathrm{mg} \\
\text { per week or as } \\
\text { tolerated plus } \\
\text { Golimumab } 50 \mathrm{mg} \\
\text { administrated every } 4 \\
\text { weeks }\end{array}$ & $\begin{array}{l}\text { Methotrexate } 25 \mathrm{mg} \\
\text { per week or as } \\
\text { tolerated plus } \\
\text { placebo prefilled } \\
\text { syringes } \\
\text { administrated every } 4 \\
\text { weeks }\end{array}$ & 24 & 24 & $\begin{array}{l}\text { Dactylitis Severity Score (DSS) } \\
\text { DSS20, } 50 \text { or } 70, \text { Leeds } \\
\text { Dactylitis Index (LDI) LDI20, } 50 \text { or } \\
70 \text {, Enthesitis Index (LEI). }\end{array}$ & $\begin{array}{l}\text { Percentage of patients } \\
\text { with AEs. }\end{array}$ & $2 \mathrm{~b}$ \\
\hline 18. & Lee et al. (32) & $\begin{array}{l}\text { Randomized, } \\
\text { open labeled } \\
\text { trial }\end{array}$ & 60 & $\geq 10 \% \mathrm{BSA}$ or PASI $\geq 10$ & $\begin{array}{l}\text { Etanercept } 25 \mathrm{mg} \\
\text { biweekly plus acitretin } \\
10 \mathrm{mg} \text { twice daily for } \\
24 \text { weeks }\end{array}$ & $\begin{array}{l}\text { (i) Etanercept } 50 \mathrm{mg} \\
\text { biweekly for } 12 \text { weeks } \\
\text { followed by } \\
\text { etanercept } 25 \mathrm{mg} \\
\text { biweekly for } 12 \\
\text { weeks; (ii) Acitretin } \\
10 \mathrm{mg} \text { BID for } 24 \\
\text { weeks. }\end{array}$ & 24 & 24 & $\begin{array}{l}\text { PASI 75, PASI 50, } \\
\text { clear/almost-clear by PGA }\end{array}$ & $\begin{array}{l}\text { Percentage of patients } \\
\text { with AEs }\end{array}$ & $2 \mathrm{~b}$ \\
\hline
\end{tabular}


TABLE 1 | Continued

\begin{tabular}{|c|c|c|c|c|c|c|c|c|c|c|c|}
\hline \multirow[t]{2}{*}{ S no. } & \multirow[t]{2}{*}{ References } & \multirow[t]{2}{*}{ Study design } & \multirow{2}{*}{$\begin{array}{l}\text { No of } \\
\text { patients }\end{array}$} & \multirow{2}{*}{$\begin{array}{l}\text { Baseline disease } \\
\text { severity }\end{array}$} & \multirow[t]{2}{*}{ Intervention } & \multirow[t]{2}{*}{ Control group(s) } & \multirow{2}{*}{$\begin{array}{l}\text { Study length } \\
\text { (weeks) }\end{array}$} & \multirow{2}{*}{$\begin{array}{l}\text { Follow up } \\
\text { (weeks) }\end{array}$} & \multicolumn{2}{|c|}{ Outcome measures used in study analysis } & \multirow[t]{2}{*}{ LoE } \\
\hline & & & & & & & & & Efficacy & Safety & \\
\hline 19. & $\begin{array}{l}\text { Gisondi et al. } \\
\text { (33) }\end{array}$ & $\begin{array}{l}\text { Randomized, } \\
\text { controlled, } \\
\text { investigator- } \\
\text { blinded pilot } \\
\text { trial }\end{array}$ & 60 & $\geq 10 \% \mathrm{BSA}$ or $\mathrm{PASI} \geq 10$ & $\begin{array}{l}\text { Etanercept } 25 \mathrm{mg} \\
\text { once weekly plus oral } \\
\text { acitretin } 0.4 \mathrm{mg} \text { per } \\
\mathrm{kg} \text { per day daily. }\end{array}$ & $\begin{array}{l}\text { Etanercept } 25 \mathrm{mg} \\
\text { twice weekly } \\
\text { subcutaneously; (ii) } \\
\text { Acitretin } 0.4 \mathrm{mg} \text { per } \\
\text { kg per day daily in a } \\
\text { single oral dose; and }\end{array}$ & 24 & 24 & $\begin{array}{l}\text { PASI } 75, \text { PASI } 50 \text { and mean BSA } \\
\text { reduction at week } 24\end{array}$ & $\begin{array}{l}\text { Percentage of patients } \\
\text { with AEs }\end{array}$ & $2 \mathrm{~b}$ \\
\hline 20. & $\begin{array}{l}\text { van Bezooijen } \\
\text { et al. (34) }\end{array}$ & $\begin{array}{l}\text { Randomized } \\
\text { controlled trial }\end{array}$ & 33 & $\mathrm{PASI} \geq 10$ & $\begin{array}{l}\text { Oral fumarates up to } \\
4 \times 215 \mathrm{mg} \text { plus } \\
\text { Etanercept } 2 \times 50 \\
\mathrm{mg} / \text { week for } 12 \\
\text { weeks followed by } 1 \\
\times 50 \mathrm{mg} \text { weekly from } \\
\text { week } 12 \text { onwards }\end{array}$ & $\begin{array}{l}\text { Etanercept at } 2 \times 50 \\
\mathrm{mg} / \text { week for } 12 \\
\text { weeks followed by } \\
\text { etanercept to } 1 \times \\
50 \mathrm{mg} \text { weekly from } \\
\text { week } 12 \text { onwards }\end{array}$ & 48 & 48 & $\begin{array}{l}\text { PASI 75, PGA clear or almost } \\
\text { clear }\end{array}$ & $\begin{array}{l}\text { Percentage of patients } \\
\text { with AEs }\end{array}$ & $2 \mathrm{~b}$ \\
\hline 21. & $\begin{array}{l}\text { Tzaneva et al. } \\
\text { (35) }\end{array}$ & $\begin{array}{l}\text { Open-label } \\
\text { randomized trial }\end{array}$ & 30 & $\geq 10 \% \mathrm{BSA}$ or $\mathrm{PASI} \geq 10$ & $\begin{array}{l}\text { Accelerated FAE } \\
\text { dosing scheme with } \\
\text { NB-UVB thrice weekly }\end{array}$ & Accelerated FAE & 26 & 26 & $\begin{array}{l}\text { Mean PASI reduction, PASI 75, } \\
\text { Mean, absolute and relative DLQI } \\
\text { reduction }\end{array}$ & $\begin{array}{l}\text { Percentage of patients } \\
\text { with AEs }\end{array}$ & $2 \mathrm{~b}$ \\
\hline 22. & $\begin{array}{l}\text { Prystowsky } \\
\text { et al. (36) }\end{array}$ & $\begin{array}{l}\text { Randomized, } \\
\text { single blinded, } \\
\text { placebo- } \\
\text { controlled } \\
\text { trial }\end{array}$ & 19 & $>20 \%$ BSA & $\begin{array}{l}\text { Calcitriol } 0.5-2.0 \mu \mathrm{g} \\
\text { per day plus NB-UVB } \\
\text { four times weekly }\end{array}$ & $\begin{array}{l}\text { Placebo plus NB-UVB } \\
\text { four times weekly }\end{array}$ & 5 & NR & $\begin{array}{l}\text { Mean change in PASI (scale, } \\
0-16 \text { ) }\end{array}$ & NR & $2 \mathrm{~b}$ \\
\hline 23. & $\begin{array}{l}\text { Ezquerra et al. } \\
(37)\end{array}$ & $\begin{array}{l}\text { Open-label } \\
\text { randomized trial }\end{array}$ & 40 & $\mathrm{PASI} \geq 15$ & $\begin{array}{l}\text { Acitretin at } 25 \mathrm{mg} \text { per } \\
\text { day plus calcitriol } \\
0.25 \mu \mathrm{g} \text { per day }\end{array}$ & $\begin{array}{l}\text { Acitretin at } 25 \mathrm{mg} \text { per } \\
\text { day }\end{array}$ & 12 & NR & Mean change in PASI & $\begin{array}{l}\text { Percentage of patients } \\
\text { with AEs }\end{array}$ & $2 \mathrm{~b}$ \\
\hline 24. & Mittal et al. (38) & $\begin{array}{l}\text { Randomized, } \\
\text { double-blind, } \\
\text { placebo- } \\
\text { controlled } \\
\text { trial }\end{array}$ & 41 & $>20 \% \mathrm{BSA}$ & $\begin{array}{l}\text { Acitretin at } 25 \mathrm{mg} \text { per } \\
\text { day plus pioglitazone, } \\
\text { Hydrochloride at } \\
15 \mathrm{mg} \text { per day }\end{array}$ & $\begin{array}{l}\text { Acitretin at } 25 \mathrm{mg} \text { per } \\
\text { day plus placebo }\end{array}$ & 12 & 12 & $\begin{array}{l}\text { PASI } 75 \text {, PGA of clear or almost } \\
\text { clear, mean change in PASI, } \\
\text { withdrawal because of lack of } \\
\text { efficacy }\end{array}$ & $\begin{array}{l}\text { Percentage of patients } \\
\text { with AEs, withdrawal } \\
\text { because of AEs }\end{array}$ & $2 \mathrm{~b}$ \\
\hline 25. & $\begin{array}{l}\text { el-Mofty et al. } \\
\text { (39) }\end{array}$ & $\begin{array}{l}\text { Randomized } \\
\text { trial, unclear } \\
\text { masking }\end{array}$ & 16 & $>25 \%$ BSA & $\begin{array}{l}\text { Sulfasalazine, } 2 \text { gm } \\
\text { per day plus } \\
\text { Pentoxifylline } \\
1,200 \mathrm{mg} \text { per day }\end{array}$ & $\begin{array}{l}\text { Methotrexate, } 25 \mathrm{mg} \\
\text { per week }\end{array}$ & 8 & NR & $\begin{array}{l}\text { Mean change in PASI, } \\
\text { Withdrawal because of lack of } \\
\text { efficacy }\end{array}$ & $\begin{array}{l}\text { Percentage of patients } \\
\text { with AEs }\end{array}$ & $2 \mathrm{~b}$ \\
\hline
\end{tabular}

PUVA, Psoralen and Ultra Violet A; UVB, Ultra Violet B; NB-UVB, Narrow Band Ultra Violet B; BSA, Body Surface Area; PASI, Psoriasis Area and Severity Index; AE, Adverse Events; SAE, Serious Adverse Events; BMI, Body Mass Index; DLQI, Dermatology Life Quality Index; PGA, Physician's Global Assessment; sPGA, static Physician's Global Assessment; PtGA, Patient's Global Assessment; ACR 20/50/70, American College of Rheumatology 20/50//70; MDA response, Minimal Disease Activity response; LDI 20/50/70, Leeds Dactylitis Index 20/50/70; EULAR response, European League Against Rheumatism response; DAS28 scores, Disease Activity Score in 28 joints; LSI, Leeds Enthesitis Index. 
TABLE 2 | Summary of levels of evidence and strength of recommendations.

\begin{tabular}{|c|c|c|c|}
\hline S. no. & Drug combinations & $\begin{array}{l}\text { Highest levels } \\
\text { of evidence on } \\
\text { efficacy }\end{array}$ & $\begin{array}{l}\text { Recommendations } \\
\text { for combination } \\
\text { on basis of } \\
\text { evidence }\end{array}$ \\
\hline 1 & UVB/PUVA + Acitretin $(15,16)$ & $2 b$ & $\mathrm{~B}$ \\
\hline 2 & Etanercept + NB-UVB (17-20) & $1 b, 2 b$ & $A$ \\
\hline 3 & Adalimumab + NB-UVB $(40,41)$ & $2 b, 4$ & $\mathrm{~B}$ \\
\hline 4 & Ustekinumab + NB-UVB (21) & $2 b$ & $\mathrm{~B}$ \\
\hline 5 & Methotrexate + NB-UVB (22-24) & $1 b, 2 b$ & A \\
\hline 6 & $\begin{array}{l}\text { Etanercept + Methotrexate } \\
(25-28)\end{array}$ & $1 b, 2 b$ & $A$ \\
\hline 7 & Infliximab + Methotrexate (29) & $2 b$ & $\mathrm{~B}$ \\
\hline 8 & $\begin{array}{l}\text { Golimumab + Methotrexate } \\
(30,31)\end{array}$ & $2 b$ & $\mathrm{~B}$ \\
\hline 9 & Etanercept + Acitretin $(32,33)$ & $2 b$ & $B$ \\
\hline 10 & Apremilast + NB-UVB (42) & 4 & C \\
\hline 11 & $\begin{array}{l}\text { Apremilast + Secukinumab } \\
(43,44)\end{array}$ & 4 & C \\
\hline 12 & Etanercept + Fumarates (34) & $2 b$ & $\mathrm{~B}$ \\
\hline 13 & Fumarates + NB-UVB (35) & $2 b$ & $\mathrm{~B}$ \\
\hline 14 & Calcitriol (oral) + Acitretin (37) & $2 b$ & $\mathrm{~B}$ \\
\hline 15 & Hydroxyurea + Acitretin (45) & 4 & C \\
\hline
\end{tabular}

Levels of evidence: 1a, Systematic review of (homogeneous) RCTs; 1b, Individual RCTs (with narrow confidence intervals); 2a, Systematic review of (homogeneous) cohort studies of "exposed" and "unexposed" subjects; 2b, Individual cohort study/low-quality RCTs; 3a, Systematic review of (homogeneous) case-control studies; 3b, Individual case-control studies; 4, Case series, low-quality cohort or case-control studies; 5, Expert opinions based on non-systematic reviews of results or mechanistic studies.

Strength of recommendations: A, Good evidence to support a recommendation for use; $B$, Moderate evidence to support a recommendation for use; $C$, Poor evidence to support a recommendation

etanercept therapy. Calzavara-Pinton et al. (19) inferred that the combination is more effective than each therapy alone in the treatment of moderate-to-severe plaque psoriasis, and is welltolerated. In an intra-individual RCT by Gambichler et al. (20) $(n=14)$ the relative M-PASI (modified-PASI) reduction of etanercept alone treated sites after 6-weeks was $53.7 \pm 36.9 \%$, whereas etanercept plus NB-UVB combination treated sites resulted in a significantly higher relative M-PASI reduction of $64.7 \pm 27.8 \%(P=0.011,95 \%$ CI -19 to $-3 \%)$ concluding that etanercept combined with NB-UVB is more effective than etanercept monotherapy at 6 weeks. Similarly, in an another intra-individual RCT by Wolf et al. (40) consisting of 04 participants who were followed up for 06 weeks concluded that adding thrice weekly NB-UVB to $40 \mathrm{mg}$ bi-weekly adalimumab reduced mean PASI from 14.8 to 2.0 on UV-irradiated body halves vs. 6.9 on non-irradiated body halves (95\% confidence interval, 0.4-9.4) accelerating the clearance of psoriatic lesions with no significant adverse effects. Bagel (41) performed a 24week single-arm open-label study in 20 adults with moderate to severe psoriasis who received bi-weekly adalimumab $40 \mathrm{mg}$ and thrice weekly NB-UVB phototherapy for 12 weeks and followed up for another 12 weeks. The mean baseline scores of patients were 17.0 for PASI, 21.2 for BSA (Body Surface Area) and 3.5 for
PGA (Physicians Global Assessment). At the end of treatment at week 12, 19 (95\%) patients achieved PASI-75, 15 (75\%) PASI-90 and 11 (55\%) achieved PASI-100. Seventeen (85\%) were clear or almost clear (PGA score $=1$ ). Mean baseline PASI, BSA, and PGA scores improved by 95,93 , and $80 \%$, respectively. Moreover, the improvement was sustained through the end of follow up period at week 24 without any serious adverse events. Although none of the studies combining TNF $\alpha$ blockers and NB-UVB reported any major adverse effects, concerns were shown regarding the long-term effects of combining TNF $\alpha$ blockers with NB-UVBespecially malignancy.

\section{Recommendation}

As the implication of malignancy in treatment with TNF- $\alpha$ blockers alone or in combination with NB-UVB complex with levels of TNF- $\alpha$ having varied effects on tumoral growth, (51) we recommend to restrict this highly effective combination for short duration up to 24 weeks, to obtain a quicker response and to avoid long-term complications (52-54). European Academy of Dermatology and Venereology (EADV) guidelines on management of psoriasis mention that $\mathrm{TNF} \alpha$ blockers and NB-UVB may or may not be combined and it is not as strict a contraindication as cyclosporine with NB-UVB (55).

\section{NB-UVB and IL12/23 Inhibitor}

There is only a single intra-individual RCT combining injection ustekinumab at 45/90 mg 4 weeks apart and thrice weekly 311nm UVB by Wolf et al. (21) in 10 patients. At baseline, the mean PASI was similar in both irradiated and unirradiated body halves (13.6 vs. 13.3). At 6 weeks, PASI was significantly lower on irradiated body halves ( 2.5 vs. 6.1), (95\% confidence interval 1.3-5). PASI 75 was achieved significantly more often on UVirradiated body halves than on un-irradiated ones [7/9 patients (78\%) vs. 1/9 (11\%)]. They concluded that treatment with NBUVB accelerates the clearance of psoriatic lesions at week 6 as well as at week 12 in ustekinumab-treated patients without increase in incidence of severe adverse effects.

\section{Recommendation}

No specific recommendation could be offered as there is limited review of this combination. However, in patients on ustekinumab with a poor response NB-UVB may be added as it has a good safety profile.

\section{NB-UVB and Methotrexate}

03 studies combining methotrexate and NB-UVB met the criteria to be included in our review- Mahajan et al. (22), Asawanonda et al. (23), and Al-Hamamy et al. (24). Mahajan et al. (22) combined oral methotrexate at $0.5 \mathrm{mg} / \mathrm{kg}$ once weekly [maximum of $30 \mathrm{mg} /$ week and thrice weekly NB-UVB and compared it with placebo plus NB-UVB for a duration of 12 weeks. PASI 75 was attained in 19/20 patients in the combination group versus $14 / 20$ patients in NB-UVB plus placebo group $(p=0.04)$ ]. PASI 75 was achieved in $7.57 \pm 3.09$ weeks $(4-$ $16)$ in the combination group and $11.42 \pm 4.98$ weeks $(6-20)$ in NB-UVB + placebo group $(p<0.006)$. The mean number of NB-UVB sessions to which the patients were exposed were 
$17.47 \pm 6.62(10-35)$ in the combination group and 35.72 $\pm 17.05(16-6)$ in NB-UVB + placebo group $(p<0.0001)$. Mean NBUVB dose for achieving PASI 75 was $9.14 \pm 5.39$ $\mathrm{J} / \mathrm{cm}^{2}(3.34-20.84)$ in the combination group as compared with $25.99 \pm 18.55 \mathrm{~J} / \mathrm{cm}^{2}$ in NB-UVB + placebo group $(p<0.001)$. Asawanonda et al. (23) showed that the median time to clear psoriasis in the former group was 4 weeks, which was significantly less than that the latter. Ten of 11 patients on combination of methotrexate and NBUVB achieved PASI 90 compared with only $5 / 13$ in the placebo/ NB-UVB group $(p<0.0001)$. The mean cumulative dose in methotrexate/NB-UVB group was $26.92 \pm$ $15.54 \mathrm{~J} / \mathrm{cm}^{2}$, as compared to $59.25 \pm 16.71 \mathrm{~J} / \mathrm{cm}^{2}(p=0.002)$ in the placebo/NBUVB group. Al-Hamamy et al. (24) compared the combination of methotrexate with NB-UVB, methotrexate alone and NB-UVB alone and found no statistically significant difference in the number of patients achieving PASI 90 between the three groups in six months of treatment. However, the mean number of weeks required for achieving clearance was $6.11 \pm$ 1.28 weeks in combination group and $11.42 \pm 2.36$ weeks in NBUVB group, while $20.87 \pm 4.21$ weeks in methotrexate group ( $p$ $<0.0001)$. The mean number of NBUVB sessions to which the patients were exposed was $17.86 \pm 3.74$ in combination group and $33.51 \pm 6.90$ in NB-UVB group $(p<0.0001)$. The mean total cumulative dose of NBUVB phototherapy for achieving clearance was $12.13 \pm 4.02 \mathrm{~J} / \mathrm{cm}^{2}$ in the combination group; compared with $34.48 \pm 13.13 \mathrm{~J} / \mathrm{cm}^{2}$ in NB-UVB group $(p<0.0001)$. All $03 \mathrm{RCTs}$ combining methotrexate with NB-UVB concluded that the mean time to achieve reduction in PASI 75 was significantly less in the combined group as against those treated only with NB-UVB and addition of methotrexate to NB-UVB rapidly clears psoriatic lesions without any significant adverse effects.

\section{Recommendation}

We recommend combining NB-UVB with methotrexate for faster clearance of lesions. However, either may be discontinued after achieving PASI 75 and the other continued for maintenance therapy the duration of which shall be dictated by the disease burden.

\section{TNF $\alpha$ Inhibitors and Methotrexate}

The following RCTs combining TNF $\alpha$ blockers with methotrexate met the inclusion criteria- Zachariae et al. (25), Gottlieb et al. (26), Yu et al. (27), Mease et al. (28), Baranauskaite et al. (29), van Mens et al. (30), and VieiraSousa et al. (31) who compared the combination of $\mathrm{TNF} \alpha$ blockers with methotrexate with either TNF $\alpha$ blockers alone or methotrexate alone. Zachariae et al. (25) randomized 60 patients who were already on methotrexate for at least 03 months into two groups receiving etanercept-methotrexate combination and etanercept with tapering and stopping methotrexate and noted significantly more number of patients achieving PASI 75 as well as significantly lower mean PASI scores at both 12 and 24 weeks in the combination group compared with etanercept alone with similar AEs for both groups, an effect that was maintained until the end of the study. Gottlieb et al. (26) studied 478 patients combining weekly methotrexate to patients who were already on etanercept since 24 weeks to the treatment group against placebo in control group. The percentage of patients achieving PASI 75 was significantly higher at week 24 for the combination therapy group (77.3\%) compared with the monotherapy group (60.3\%; $p<0.0001)$. Overall, $74.9 \%$ of patients in the combination group experienced AEs compared with $59.8 \%$ in the monotherapy group. Withdrawals due to AEs were infrequent in both groups [combination, $n$ $=10(4.2 \%)$; monotherapy, $n=6(2.5 \%)]$, and none of the AEs leading to withdrawal was considered to be serious or infectious. They concluded that addition of methotrexate to etanercept was more effective than etanercept monotherapy in patients with moderate to severe plaque psoriasis with acceptable tolerability. Yu et al. (27) compared similar treatment arms as above but started administering the combination from baseline and followed up subjects for 24 weeks. They found no significant change in the PASI score from baseline to 24 weeks. However, both sPGA (static Physician's Global Assessment) and PtGA (Patient's Global Assessment) scores were significant $(p<0.05)$. Adverse effects were reported in $60 \%$ of patients in the combination group and in $33 \%$ in the monotherapy group. None of the adverse effects were serious enough to discontinue treatment. Mease et al. (28) performed a triple arm study consisting of 851 patients of psoriatic arthropathy randomized to oral methotrexate $20 \mathrm{mg}$ plus placebo weekly, etanercept $50 \mathrm{mg}$ plus placebo weekly, or etanercept $50 \mathrm{mg}$ plus oral methotrexate $20 \mathrm{mg}$ weekly. ACR20 (American College of Rheumatology 20) criteria and MDA (Minimal Disease Activity) responses at week 24 were significantly greater for etanercept monotherapy vs. methotrexate monotherapy (ACR20: 60.9 vs. $50.7 \%$ [ $p=0.029]$; MDA: 35.9 vs. $22.9 \%[p=0.005])$ and for combination therapy vs. methotrexate monotherapy (ACR20: 65.0 vs. $50.7 \%$ [ $p=0.005]$; MDA: 35.7 vs. $22.9 \%[p=0.005])$. Many patients in this trial had a moderate to severe level of psoriasis as assessed by BSA (Body Surface Area). Results from the dermatologic endpoints showed that etanercept and methotrexate had good efficacy, with a suggestion that the combination arm had slightly greater efficacy than either of the monotherapy arms for improved BSA. They concluded that etanercept monotherapy and combination therapy showed greater efficacy than methotrexate monotherapy in ACR, MDA, and BSA responses and radiographic progression. However, combining methotrexate and etanercept did not improve etanercept efficacy in either PsA or psoriasis. Baranauskaite et al. (29) combined infliximab at $5 \mathrm{mg}$ per $\mathrm{kg}$ infusions at $0,2,6$, and 14 weeks and methotrexate at $15 \mathrm{mg}$ per week vs. methotrexate alone for a period of 16 weeks. $86.3 \%$ of patients receiving combination and $66.7 \%$ of those receiving methotrexate alone achieved an ACR20 response $(p<0.02)$. While $97.1 \%$ of patients receiving infliximab plus methotrexate achieved PASI 75 , the figure was $54.3 \%$ in patients receiving methotrexate alone ( $p$ $<0.0001)$. They demonstrated significantly greater ACR 20 response rates and PASI 75 improvement in the combination group and was generally well-tolerated. A double-blind RCT measuring end points in psoriatic arthritis by van Mens et al. (30) studied combination of methotrexate $15-25 \mathrm{mg}$ per week and subcutaneous injections of golimumab at $50 \mathrm{mg}$ per month with that of methotrexate and placebo and found that 
Disease Activity Score (DAS) remission at week 22 was almost doubled $(21 / 26 ; 81 \%)$ in methotrexate plus golimumab group vs. methotrexate alone $(10 / 24 ; 42 \%)(p=0.004)$. Also the patients belonging to the combination group reached an MDA (Minimal Disease Activity) in 21/26 (81\%) vs. 7/24 (29\%) in the methotrexate arm $(p<0.001)$. An ACR 20/50/70 response was achieved by, respectively, 85,81 , and $58 \%$ in the combination arm vs. 58,33 , and $13 \%$ in the methotrexate arm $(p=0.039$, $p=0.001$, and $p=0.001$, respectively). The most frequent adverse effect was nausea and occurred in similar incidences in both treatment arms and considered to be treatment related but was not severe enough to discontinue treatment. Likewise, a double-blind RCT by Vieira-Sousa et al. (31) comparing similar doses of golimumab plus methotrexate vs. placebo plus methotrexate in dactylitis in psoriatic arthropathy concluded that the combination of golimumab and methotrexate was superior to methotrexate alone in reducing Dactylitis Severity Score (DSS) and Leeds Dactylitis Index (LDI) with comparable incidence of adverse effects between treatment arms. All patients had active dactylitis at baseline, with a median baseline DSS of 6 in both arms. The patients treated with golimumab/methotrexate exhibited significantly greater improvements by DSS at week 24 (median change of 5) relative to the placebo/methotrexate group (median change of 2$)(p=0.026)$, and as early as 12 weeks $(p=0.004)$. The proportion of DSS50 (Dactylitis Severity Score 50) and DSS70 (Dactylitis Severity Score 70) responders at week 24 were also significantly higher for patients treated with golimumab/methotrexate (DSS50: $p=0.005$, DSS70: $p=$ 0.010) Endpoints to measure cutaneous efficacy like PASI, BSA and skin-related quality of life (Dermatology Life Quality Index) improved in both groups at week 24 but difference in both treatment groups was not significant. 102 adverse events were reported during study period, with similar incidence between the treatment arms and mostly of mild to moderate severity. According to systematic review by Hsu et al. (56), there are 06 studies measuring anti-drug antibodies in etanercept and its possible effect on drug efficacy- they found the prevalence of anti- etanercept antibodies (AEA) ranging from 0 (57) to $18.3 \%$ (58) in psoriasis, and none of which had significant effect on treatment efficacy (56). Similarly, 10 studies proved prevalence of anti-infliximab antibodies (AIA) ranging from 5.4 (59) to $43.6 \%$ (60) with most of these studies showing significant decreased mean PASI scores and greater loss of clinical response when compared to AIA-negative patients (56). A study by Adisen et al. (61) with five patients of psoriasis who developed AIA, determined that AIA positivity disappeared after 8 weeks of combined methotrexate pulse therapy, ranging from 5 to 15 mg/week. Six studies assessed for Anti-Ustekinumab Antibody (AUA) formation in patients with moderate-to severe psoriasis showed ranges from 3.8 (62) to $5.4 \%$ (63) in psoriasis (56). But their clinical significance on treatment response is yet to be evaluated (56).

\section{Recommendation}

We recommend combining $\mathrm{TNF} \alpha$ blockers with methotrexate in moderate to severe psoriatic disease especially while using infliximab. Poor response to etanercept alone at lower doses as elaborated below necessitates an additional drug, methotrexate being a good option.

\section{TNF $\alpha$ Inhibitors With Cyclosporine}

Atzeni et al. (64) who performed an RCT comparing etanercept plus cyclosporine with etanercept plus methotrexate show similar efficacy in reducing DAS28 (Disease Activity Score 28 ) in patients with moderate/severe psoriatic arthropathy and peripheral arthritis, but former combination was more efficacious in reducing psoriatic skin involvement. PASI 50 and PASI 75 scores were achieved by 88 and 53\%, respectively, in the patients in etanercept plus cyclosporin group, and 73 and $32 \%$, respectively, in the patients in etanercept plus methotrexate group $(p<0.05)$. There was no significant difference in serious adverse events between the two treatment groups.

\section{Recommendation}

We recommend $\mathrm{TNF} \alpha$ blockers with cyclosporine in moderate to severe psoriasis with arthropathy for rapid remission, however side effects limit the duration of treatment with cyclosporine and sequential therapy with methotrexate is recommended.

\section{TNF $\alpha$ Inhibitors With Acitretin}

Lee et al. (32) randomized 60 subjects into three treatment armsETN-ETN (etanercept-etanercept), ETN-ACT (etanerceptacitretin), and ACT (acitretin). The median time to achieve PASI 75 for patients in the ETN-ETN arm was 126 days vs. 146 days for patients in the ETN-ACT arm. The median time to achieve PASI 50 was same in ETN-ETN and ETN-ACT arms (56 days) and much shorter than for patients in ACT arm (126 days). The difference was statistically significant among the three treatment arms (PASI 75: $p=0.0448$ and PASI 50: $p=0.0033$ ). Lee et al. (32) proved that the combination is more effective than acitretin alone without increase in adverse effects. In another study with similar treatment arms Gisondi et al. (33) randomized 60 patients into three groups to receive etanercept $25 \mathrm{mg}$ twice weekly; acitretin $0.4 \mathrm{mg}$ per kg daily; and etanercept $25 \mathrm{mg}$ once weekly plus oral acitretin $0.4 \mathrm{mg}$ per $\mathrm{kg}$ daily. PASI 75 response at week 24, was achieved by 10 of 22 patients (45\%) in the etanercept group, six of $20(30 \%)$ in the acitretin group and eight of $18(44 \%)$ patients with etanercept plus acitretin group $(P=$ 0.001 for both etanercept groups compared with acitretin alone). PASI 50 response at week 24 too showed similar significant results $(P=0.001$ for both etanercept groups compared with acitretin alone).

\section{Recommendation}

Etanercept $25 \mathrm{mg}$ twice weekly with acitretin is a superior option to acitretin alone. We recommend addition of acitretin to etanercept dose of $25 \mathrm{mg}$ twice weekly before considering a higher dose of etanercept $50 \mathrm{mg}$ twice weekly.

\section{Apremilast Combinations}

Apremilast, a PDE4 inhibitor has minimal immunosuppressive effects when compared to biologics. There are no RCTs combining apremilast with any other drug. However, case series and retrospective studies have suggested that combination of apremilast with other drugs and biologics like methotrexate, 
acitretin, cyclosporin, secukinumab, etanercept, adalimumab, ixekizumab, and ustekinumab have been effective, look promising and may be exercised to reduce adverse effects of either of two.

In an open-labeled prospective study combining apremilast $30 \mathrm{mg}$ twice daily and increasing doses of NB-UVB three times per week for 12 weeks. $73 \%$ (16 of 22 completers) achieved a PASI 75 response at week 12 . The most commonly reported adverse events were mild and moderate first-degree burns related to NB-UVB ( $n=11$ [38\%] patients). Bagel et al. (42) concluded that the combination provided a new treatment option without any increased adverse effects. Both Sacchelli et al. (43) and De et al. (44) published case series and case reports combining apremilast with secukinumab and found improvement in PASI scores. Metyas et al. (65) and Takamura et al. (66) performed retrospective studies reporting the efficacy of apremilast in combination with any other biologics and inferred that apremilast can be safely combined with all biologic agents in patients with plaque psoriasis or psoriatic arthritis not responding adequately to biologics alone. Another retrospective study by AbuHilal et al. (67) studied the combination of apremilast with other biologics as well as conventional drugs like methotrexate, acitretin, cyclosporine with similar conclusion.

\section{Recommendation}

We recommend apremilast $30 \mathrm{mg}$ twice daily and NB-UVB as a combination modality not responding or minimally responding to either of the two. The combination of apremilast and a biologic may be a safe, useful treatment option for managing patients with psoriasis showing biologic fatigue, but not as a routine. However, large scale studies with higher level of evidence like RCTs are needed in future.

\section{Miscellaneous Combination Therapies}

RCTs combining less used unconventional drugs in psoriasis included in this review dealt with fumaric acid esters (FAEs) calcitriol, sulfasalazine, pentoxifylline, and pioglitazone with conventional modes of therapy. An exploratory RCT by Bezooijen et al. (34) combining etanercept $50 \mathrm{mg}$ twice weekly for 12 weeks followed by once weekly for another 12 weeks with oral fumarates $215 \mathrm{mg}$ four times daily for the whole period vs. etanercept alone found out that the reduction in PASI score per week for the combination therapy was $5.97 \%$ (95\% confidence interval, CI: 5.08-6.85) and in the monotherapy group $4.76 \%$ (95\% CI: 3.57-5.93; $p=0.11$ ). They concluded that combination therapy caused quicker improvement in PASI 75 in first 24 weeks although difference in the PASI score between the two groups was statistically insignificant but with satisfactory tolerability. In an another RCT by Tzaneva et al. (35), an increasing dose of FAEs was combined with NB-UVB. At 26 weeks of treatment, the median baseline PASI of 15.4 [interquartile range (IQR) 11.7-21.0] was reduced to 2.8 (IQR 1.6-4.8) in the combination group and from 14.0 (IQR 12.515.1 ) to 9.0 (IQR 6.5-12.1) in the FAE group, respectively. The mean absolute and relative reduction in PASI was significantly greater in the combination group (10 and 69\%) compared with patients receiving only FAE (5 and 36\%) $(p=0.016)$. Side-effects related to FAE were mainly mild gastrointestinal complaints reported by $12 / 16$ patients $(75 \%)$ in the monotherapy group and $3 / 14$ patients $(21 \%)$ in the combination group. These were abdominal pain, nausea, flatulence, diarrhea that occurred at the beginning of treatment, were dose-dependent and improved after a temporary dose reduction. They found an accelerated as well as augmented response improving the quality of life in the patients with combination therapy as compared with fumaric acid esters monotherapy with no increase in adverse effects in the combination group.

A single blinded, placebo-controlled trial combining calcitriol 0.5-2.0 $\mu \mathrm{g}$ per day plus NB-UVB against NB-UVB alone by Prystowsky et al. (36) concluded that there was no added benefit to treatment when oral calcitriol was administered with phototherapy. Our search yielded only a single RCT combining acitretin and calcitriol- Ezquerra et al. (37) who compared the combination with acitretin alone. Initial PASI of 26.90 reduced to 13.3 in acitretin alone group; whereas it reduced from 28.35 to 10.3 in acitretin+calcitriol combination group which was statistically significant $(p<0.05)$. A double-blind RCT by Mittal et al. (38) compared combination of acitretin plus pioglitazone hydrochloride with acitretin alone. The percentage of reduction in the PASI score from baseline to 12 weeks of treatment was $64.2 \%$ (95\% CI, 49.2-79.3\%) in the combination group compared with $51.7 \%(95 \% \mathrm{CI}, 38.7-64.7 \%)$ in the acitretin plus placebo group $(p=0.04)$. The adverse effects in both the groups were mild to moderate and were comparable. el-Mofty et al. (39) conducted a quadri-armed RCT comparing the combination of sulfasalazine and pentoxifylline to methotrexate alone (active control group), sulfasalazine alone and pentoxifylline alone and concluded that combination of sulfasalazine and pentoxifylline though effective than when used alone, is not as effective as methotrexate, may be promising and tried because they present as safer and well-tolerated alternatives to methotrexate. There are no RCTs on hydroxyurea in psoriasis. Hydroxyurea becomes one of the drugs of choice in settings of psoriasis in HIV, where not only it helps in controlling psoriasis, but also in controlling viral loads especially when combined with didanosine (NRTI) $(68,69)$. In a retrospective study, Narang et al. (45) combined lower doses of hydroxyurea $1 \mathrm{~g}$ daily with acitretin $25 \mathrm{mg}$ daily for the management of refractory cases and found them to be superior to either to hydroxyurea and acitretin alone as found in previous studies. Combining acitretin with hydroxyurea may theoretically reduce the risk of non-melanoma skin cancers and actinic keratosis, which are rare but serious adverse effect of hydroxyurea $(49,70)$. Methotrexate too have been combined with hydroxyurea in lower doses (5-10 mg/week and $500 \mathrm{mg}$ per day, respectively) to good effect with no increase in adverse effects of either of the two (71). Though theoretically both the drugs may cause GI intolerance and myelosuppression, they were not found in the study.

\section{Recommendation}

We recommend combining hydroxyurea and acitretin in recalcitrant cases of psoriasis not responding to conventional stand-alone drugs. This combination also may be used in HIV 
where both the drugs do not cause immune suppression with added benefit of anti-viral action of hydroxyurea.

Combining methotrexate with hydroxurea in lower doses may help reducing dose-dependent or cumulative toxic effects of either of the two.

Our search for combinations comprising relatively newer drugs like guselkumab, tildrakizumab, certolizumab pegol, and tofacitinib yielded no results and provide gap in research with a massive potential.

\section{CONCLUSION}

Combining newer therapies with conventional ones is a promising prospect to manage difficult to treat psoriasis. Combining drugs when suited to patients needs can enhance efficacy, achieve remission, while reducing adverse effects. With available evidence, there are limited options with highest level of

\section{REFERENCES}

1. Michalek IM, Loring B, John SM. A systematic review of worldwide epidemiology of psoriasis. J Eur Acad Dermatol Venereol. (2017) 31:20512. doi: $10.1111 /$ jdv.13854

2. Arora S, Jairam A, Radhakrishnan S, Das N, Vankalakunti M. PLA2R antibody positive membranous glomerulonephropathy associated with psoriasis vulgaris. Indian J Dermatol Venereol Leprol. (2019) 85:682. doi: 10.4103/ijdvl.IJDVL_298_18

3. Gisondi P, Fostini AC, Fossà I, Girolomoni G, Targher G. Psoriasis and the metabolic syndrome. Clin Dermatol. (2018) 36:21-8. doi: 10.1016/j.clindermatol.2017.09.005

4. Rodríguez-Zúñiga MJM, García-Perdomo HA. Systematic review and metaanalysis of the association between psoriasis and metabolic syndrome. J Am Acad Dermatol. (2017) 77:657-66.e8. doi: 10.1016/j.jaad.2017.0 4.1133

5. Villani AP, Rouzaud M, Sevrain M, Barnetche T, Paul C, Richard MA, et al. Prevalence of undiagnosed psoriatic arthritis among psoriasis patients: systematic review and meta-analysis. J Am Acad Dermatol. (2015) 73:2428. doi: 10.1016/j.jaad.2015.05.001

6. Rosen CF, Mussani F, Chandran V, Eder L, Thavaneswaran A, Gladman DD. Patients with psoriatic arthritis have worse quality of life than those with psoriasis alone. Rheumatol Oxf Engl. (2012) 51:571-6. doi: 10.1093/rheumatology/ker365

7. Rech J, Sticherling M, Stoessel D, Biermann MHC, Häberle BM, Reinhardt M. Psoriatic arthritis epidemiology, comorbid disease profiles and risk factors: results from a claims database analysis. Rheumatol Adv Pract. (2020) 4:rkaa033. doi: 10.1093/rap/rkaa033

8. Gladman DD, Stafford-Brady F, Chang CH, Lewandowski K, Russell ML. Longitudinal study of clinical and radiological progression in psoriatic arthritis. J Rheumatol. (1990) 17:809-12.

9. Raychaudhuri SK, Maverakis E, Raychaudhuri SP. Diagnosis and classification of psoriasis. Autoimmun Rev. (2014) 13:4905. doi: 10.1016/j.autrev.2014.01.008

10. Heller MM, Wong JW, Nguyen TV, Lee ES, Bhutani T, Menter A, et al. Quality-of-life instruments: evaluation of the impact of psoriasis on patients. Dermatol Clin. (2012) 30:281-91, ix. doi: 10.1016/j.det.2011.11.006

11. Systemic Pharmacological Treatments for Chronic Plaque Psoriasis: A Network Meta-Analysis. Available online at: https://www.ncbi.nlm.nih.gov/ pmc/articles/PMC6486272/ (accessed April 9, 2021).

12. Polat Ekinci A, Bölük KN, Babuna Kobaner G. Secukinumab and acitretin as a combination therapy for three clinical forms of severe psoriasis in multi-drug evidence and hence recommendation. Due to a smaller number of studies in combination of drugs, research providing more high-quality evidence is required.

\section{DATA AVAILABILITY STATEMENT}

The original contributions presented in the study are included in the article/supplementary material, further inquiries can be directed to the corresponding author.

\section{AUTHOR CONTRIBUTIONS}

SA: conception and design, acquisition of data, literature search, analysis and interpretation of data, drafting the manuscript, and revising it. PD and GA: acquisition of data, literature search, analysis and interpretation of data, drafting the manuscript, and revising it. All authors contributed to the article and approved the submitted version.

refractory patients: a case series of high efficacy and safety profile. Dermatol Ther. (2020) e14704. doi: 10.1111/dth.14704

13. Kim WB, Jerome D, Yeung J. Diagnosis and management of psoriasis. Can Fam Physician Med Fam Can. (2017) 63:278-85.

14. Rønholt K, Iversen L. Old and new biological therapies for psoriasis. Int $\mathrm{J} \mathrm{Mol}$ Sci. (2017) 18:2297. doi: 10.3390/ijms18112297

15. Tanew A, Guggenbichler A, Hönigsmann H, Geiger JM, Fritsch $P$. Photochemotherapy for severe psoriasis without or in combination with acitretin: a randomized, double-blind comparison study. J Am Acad Dermatol. (1991) 25:682-4. doi: 10.1016/0190-9622(91)70253-X

16. Lowe NJ, Prystowsky JH, Bourget T, Edelstein J, Nychay S, Armstrong R. Acitretin plus UVB therapy for psoriasis. Comparisons with placebo plus UVB and acitretin alone. J Am Acad Dermatol. (1991) 24:5914. doi: 10.1016/0190-9622(91)70089-K

17. Lynde CW, Gupta AK, Guenther L, Poulin Y, Levesque A, Bissonnette R. A randomized study comparing the combination of nbUVB and etanercept to etanercept monotherapy in patients with psoriasis who do not exhibit an excellent response after 12 weeks of etanercept. J Dermatol Treat. (2012) 23:261-7. doi: 10.3109/09546634.2011.607795

18. Park KK, Wu JJ, Koo J. A randomized, "head-to-head" pilot study comparing the effects of etanercept monotherapy vs. etanercept and narrowband ultraviolet B (NB-UVB) phototherapy in obese psoriasis patients. J Eur Acad Dermatol Venereol. (2013) 27:899-906. doi: 10.1111/j.1468-3083.2012.04611.x

19. Calzavara-Pinton PG, Sala R, Arisi M, Rossi MT, Venturini M, Ortel B. Synergism between narrowband ultraviolet $\mathrm{B}$ phototherapy and etanercept for the treatment of plaque-type psoriasis. Br J Dermatol. (2013) 169:1306. doi: 10.1111/bjd.12277

20. Gambichler T, Tigges C, Scola N, Weber J, Skrygan M, Bechara FG, et al. Etanercept plus narrowband ultraviolet $\mathrm{B}$ phototherapy of psoriasis is more effective than etanercept monotherapy at 6 weeks. Br J Dermatol. (2011) 164:1383-6. doi: 10.1111/j.1365-2133.2011.10358.x

21. Wolf P, Weger W, Legat FJ, Posch-Fabian T, Gruber-Wackernagel A, Inzinger $\mathrm{M}$, et al. Treatment with 311-nm ultraviolet B enhanced response of psoriatic lesions in ustekinumab-treated patients: a randomized intraindividual trial. $\mathrm{Br}$ J Dermatol. (2012) 166:147-53. doi: 10.1111/j.1365-2133.2011.10616.x

22. Mahajan R, Kaur I, Kanwar AJ. Methotrexate/narrowband UVB phototherapy combination vs. narrowband UVB phototherapy in the treatment of chronic plaque-type psoriasis-a randomized singleblinded placebo-controlled study. J Eur Acad Dermatol Venereol. (2010) 24:595-600. doi: 10.1111/j.1468-3083.2009.03486.x

23. Asawanonda P, Nateetongrungsak Y. Methotrexate plus narrowband UVB phototherapy versus narrowband UVB phototherapy alone in the treatment 
of plaque-type psoriasis: a randomized, placebo-controlled study. J Am Acad Dermatol. (2006) 54:1013-8. doi: 10.1016/j.jaad.2006.01.004

24. Al-Hamamy HR, Al-Mashhadani SA, Mustafa IN. Comparative study of the effect of narrowband ultraviolet B phototherapy plus methotrexate vs. narrowband ultraviolet $\mathrm{B}$ alone and methotrexate alone in the treatment of plaque-type psoriasis. Int J Dermatol. (2014) 53:1531-5. doi: 10.1111/ijd.12444

25. Zachariae C, Mørk NJ, Reunala T, Lorentzen H, Falk E, Karvonen SL, et al. The combination of etanercept and methotrexate increases the effectiveness of treatment in active psoriasis despite inadequate effect of methotrexate therapy. Acta Derm Venereol. (2008) 88:495-501. doi: 10.2340/00015555-0511

26. Gottlieb AB, Langley RG, Strober BE, Papp KA, Klekotka P, Creamer $\mathrm{K}$, et al. A randomized, double-blind, placebo-controlled study to evaluate the addition of methotrexate to etanercept in patients with moderate to severe plaque psoriasis. Br J Dermatol. (2012) 167:649-57. doi: 10.1111/j.1365-2133.2012.11015.x

27. Yu Q, Tong Y, Cui L, Zhang L, Gong Y, Diao H, et al. Efficacy and safety of etanercept combined plus methotrexate and comparison of expression of pro-inflammatory factors expression for the treatment of moderate-to-severe plaque psoriasis. Int Immunopharmacol. (2019) 73:44250. doi: 10.1016/j.intimp.2019.05.042

28. Mease PJ, Gladman DD, Collier DH, Ritchlin CT, Helliwell PS, Liu L, et al. Etanercept and methotrexate as monotherapy or in combination for psoriatic arthritis: primary results from a randomized, controlled phase III trial. Arthritis Rheumatol Hoboken NJ. (2019) 71:1112-24. doi: 10.1002/art.40851

29. Baranauskaite A, Raffayová H, Kungurov NV, Kubanova A, Venalis A, Helmle $\mathrm{L}$, et al. Infliximab plus methotrexate is superior to methotrexate alone in the treatment of psoriatic arthritis in methotrexate-naive patients: the RESPOND study. Ann Rheum Dis. (2012) 71:541-8. doi: 10.1136/ard.2011.152223

30. van Mens LJJ, de Jong HM, Fluri I, Nurmohamed MT, van de Sande MGH, Kok M, et al. Achieving remission in psoriatic arthritis by early initiation of TNF inhibition: a double-blind, randomised, placebo-controlled trial of golimumab plus methotrexate versus placebo plus methotrexate. Ann Rheum Dis. (2019) 78:610-6. doi: 10.1136/annrheumdis-2018-214746

31. Vieira-Sousa E, Alves P, Rodrigues AM, Teixeira F, Tavares-Costa J, Bernardo A, et al. GO-DACT: a phase $3 \mathrm{~b}$ randomised, double-blind, placebo-controlled trial of GOlimumab plus methotrexate (MTX) versus placebo plus MTX in improving DACTylitis in MTX-naive patients with psoriatic arthritis. Ann Rheum Dis. (2020) 79:490-8. doi: 10.1136/annrheumdis-2019-216500

32. Lee JH, Youn JI, Kim TY, Choi JH, Park CJ, Choe YB, et al. A multicenter, randomized, open-label pilot trial assessing the efficacy and safety of etanercept $50 \mathrm{mg}$ twice weekly followed by etanercept $25 \mathrm{mg}$ twice weekly, the combination of etanercept $25 \mathrm{mg}$ twice weekly and acitretin, and acitretin alone in patients with moderate to severe psoriasis. BMC Dermatol. (2016) 16:11. doi: 10.1186/s12895-016-0048-z

33. Gisondi P, Del Giglio M, Cotena C, Girolomoni G. Combining etanercept and acitretin in the therapy of chronic plaque psoriasis: a 24-week, randomized, controlled, investigator-blinded pilot trial. Br J Dermatol. (2008) 158:13459. doi: 10.1111/j.1365-2133.2008.08564.x

34. van Bezooijen JS, Balak DM, van Doorn MB, Looman CW, Schreurs MW, Koch BC, et al. Combination therapy of etanercept and fumarates versus etanercept monotherapy in psoriasis: a randomized exploratory study. Dermatol Basel Switz. (2016) 232:407-14. doi: 10.1159/000448135

35. Tzaneva S, Geroldinger A, Trattner H, Tanew A. Fumaric acid esters in combination with a 6-week course of narrowband ultraviolet B provides an accelerated response compared with fumaric acid esters monotherapy in patients with moderate-to-severe plaque psoriasis: a randomized prospective clinical study. Br J Dermatol. (2018) 178:682-8. doi: 10.1111/bjd.16106

36. Prystowsky JH, Muzio PJ, Sevran S, Clemens TL. Effect of UVB phototherapy and oral calcitriol (1,25-dihydroxyvitamin D3) on vitamin D photosynthesis in patients with psoriasis. J Am Acad Dermatol. (1996) 35(5 Pt 1):6905. doi: 10.1016/S0190-9622(96)90722-7

37. Ezquerra GM, Regaña MS, Millet PU. Combination of acitretin and oral calcitriol for treatment of plaque-type psoriasis. Acta Derm Venereol. (2007) 87:449-50. doi: 10.2340/00015555-0290

38. Mittal R, Malhotra S, Pandhi P, Kaur I, Dogra S. Efficacy and safety of combination Acitretin and Pioglitazone therapy in patients with moderate to severe chronic plaque-type psoriasis: a randomized, double-blind, placebo-controlled clinical trial. Arch Dermatol. (2009) 145:387-93. doi: 10.1001/archdermatol.2009.5

39. el-Mofty M, el-Darouti M, Rasheed H, Bassiouny DA, AbdelHalim M, Zaki NS, et al. Sulfasalazine and pentoxifylline in psoriasis: a possible safe alternative. J Dermatol Treat. (2011) 22:31-7. doi: 10.3109/09546630903460260

40. Wolf P, Hofer A, Weger W, Posch-Fabian T, Gruber-Wackernagel A, Legat FJ. $311 \mathrm{~nm}$ ultraviolet B-accelerated response of psoriatic lesions in adalimumabtreated patients. Photodermatol Photoimmunol Photomed. (2011) 27:1869. doi: 10.1111/j.1600-0781.2011.00594.x

41. Bagel J. Adalimumab plus narrowband ultraviolet B light phototherapy for the treatment of moderate to severe psoriasis. J Drugs Dermatol. (2011) 10:366-71.

42. Bagel J, Nelson E, Keegan BR. Apremilast and narrowband ultraviolet-B combination therapy for treating moderate-to-severe plaque psoriasis. J Drugs Dermatol. (2017) 16:957-62.

43. Sacchelli L, Patrizi A, Loi C, Bardazzi F. Combination therapy of apremilast and secukinumab in patients with moderate-to-severe, recalcitrant plaque psoriasis. Clin Exp Dermatol. (2019) 44:e243-4. doi: 10.1111/ced.14000

44. De A, Das S, Dhoot D, Sarda A. Apremilast coadministered with secukinumab for safe and effective control of psoriasis with resultant reduction of maintenance dose of the biologic. Indian J Dermatol. (2019) 64:23941. doi: 10.4103/ijd.IJD_548_18

45. Narang T, Kumar S, Handa S, Dogra S. Hydroxyurea and acitretin as a novel combination therapy in severe plaque psoriasis. Br J Dermatol. (2018) 179:1212-3. doi: 10.1111/bjd.16899

46. Olsen EA, Weed WW, Meyer CJ, Cobo LM. A double-blind, placebocontrolled trial of acitretin for the treatment of psoriasis. J Am Acad Dermatol. (1989) 21(4 Pt 1):681-6. doi: 10.1016/S0190-9622(89)70236-X

47. Lauharanta J, Geiger JM. A double-blind comparison of acitretin and etretinate in combination with bath PUVA in the treatment of extensive psoriasis. $\mathrm{Br} J$ Dermatol. (1989) 121:107-12. doi: 10.1111/j.1365-2133.1989.tb01406.x

48. Heidbreder G, Christophers E. Therapy of psoriasis with retinoid plus PUVA: clinical and histologic data. Arch Dermatol Res. (1979) 264:3317. doi: $10.1007 / \mathrm{BF} 00412661$

49. Bettoli V, Zauli S, Virgili A. Retinoids in the chemoprevention of nonmelanoma skin cancers: why, when and how. J Dermatol Treat. (2013) 24:235-7. doi: 10.3109/09546634.2012.746634

50. Bavinck JN, Tieben LM, Van der Woude FJ, Tegzess AM, Hermans J, ter Schegget J, et al. Prevention of skin cancer and reduction of keratotic skin lesions during acitretin therapy in renal transplant recipients: a double-blind, placebo-controlled study. J Clin Oncol Off J Am Soc Clin Oncol. (1995) 13:1933-8. doi: 10.1200/JCO.1995.13.8.1933

51. Montfort A, Colacios C, Levade T, Andrieu-Abadie N, Meyer N, Ségui B. The TNF paradox in cancer progression and immunotherapy. Front Immunol. (2019) 10:1818. doi: 10.3389/fimmu.2019.02515

52. Patel RV, Clark LN, Lebwohl M, Weinberg JM. Treatments for psoriasis and the risk of malignancy. J Am Acad Dermatol. (2009) 60:100117. doi: 10.1016/j.jaad.2008.12.031

53. Sheppard J, Raza K, Buckley CD. Skin cancer in psoriatic arthritis treated with anti-TNF therapy. Rheumatol Oxf Engl. (2007) 46:16223. doi: 10.1093/rheumatology/kem 214

54. Di Lernia V, Albertini G. Is antitumour necrosis factor therapy combined with ultraviolet B phototherapy safe? Br J Dermatol. (2010) 162:11478. doi: $10.1111 / \mathrm{j} .1365-2133.2010 .09663 . \mathrm{x}$

55. Nast A, Gisondi P, Ormerod AD, Saiag P, Smith C, Spuls PI, et al. European S3-Guidelines on the systemic treatment of psoriasis vulgaris-Update 2015Short version-EDF in cooperation with EADV and IPC. J Eur Acad Dermatol Venereol. (2015) 29:2277-94. doi: 10.1111/jdv.13354

56. Hsu L, Snodgrass BT, Armstrong AW. Antidrug antibodies in psoriasis: a systematic review. Br J Dermatol. (2014) 170:261-73. doi: 10.1111/bjd.12654

57. Mahil SK, Arkir Z, Richards G, Lewis CM, Barker JN, Smith CH. Predicting treatment response in psoriasis using serum levels of adalimumab and etanercept: a single-centre, cohort study. Br J Dermatol. (2013) 169:30613. doi: $10.1111 /$ bjd.12341

58. Tyring S, Gordon KB, Poulin Y, Langley RG, Gottlieb AB, Dunn $M$, et al. Long-term safety and efficacy of $50 \mathrm{mg}$ of etanercept 
twice weekly in patients with psoriasis. Arch Dermatol. (2007) 143:719-26. doi: 10.1001/archderm.143.6.719

59. Gottlieb AB, Kalb RE, Blauvelt A, Heffernan MP, Sofen HL, Ferris LK, et al. The efficacy and safety of infliximab in patients with plaque psoriasis who had an inadequate response to etanercept: results of a prospective, multicenter, open-label study. J Am Acad Dermatol. (2012) 67:642-50. doi: 10.1016/j.jaad.2011.10.020

60. Menter A, Feldman SR, Weinstein GD, Papp K, Evans R, Guzzo $\mathrm{C}$, et al. A randomized comparison of continuous vs. intermittent infliximab maintenance regimens over 1 year in the treatment of moderate-to-severe plaque psoriasis. J Am Acad Dermatol. (2007) 56:31.e115. doi: 10.1016/j.jaad.2006.07.017

61. Adişen E, Aral A, Aybay C, Gürer MA. Anti-infliximab antibody status and its relation to clinical response in psoriatic patients: a pilot study. J Dermatol. (2010) 37:708-13. doi: 10.1111/j.1346-8138.2010.00882.x

62. Griffiths CE, Strober BE, van de Kerkhof P, Ho V, Fidelus-Gort R, Yeilding $\mathrm{N}$, et al. Comparison of ustekinumab and etanercept for moderate-to-severe psoriasis. N Engl J Med. (2010) 362:118-28. doi: 10.1056/NEJMoa0810652

63. Papp KA, Langley RG, Lebwohl M, Krueger GG, Szapary P, Yeilding N, et al. Efficacy and safety of ustekinumab, a human interleukin-12/23 monoclonal antibody, in patients with psoriasis: 52-week results from a randomised, double-blind, placebo-controlled trial (PHOENIX 2). Lancet Lond Engl. (2008) 371:1675-84. doi: 10.1016/S0140-6736(08)60726-6

64. Atzeni F, Boccassini L, Antivalle M, Salaffi F, Sarzi-Puttini P. Etanercept plus ciclosporin versus etanercept plus methotrexate for maintaining clinical control over psoriatic arthritis: a randomised pilot study. Ann Rheum Dis. (2011) 70:712-4. doi: 10.1136/ard.2010.130864

65. Metyas S, Tomassian C, Messiah R, Gettas T, Chen C, Quismorio A. Combination therapy of apremilast and biologic agent as a safe option of psoriatic arthritis and psoriasis. Curr Rheumatol Rev. (2019) 15:2347. doi: 10.2174/1573397115666181130094455

66. Takamura S, Sugai S, Taguchi R, Teraki Y. Combination therapy of apremilast and biologics in patients with psoriasis showing biologic fatigue. J Dermatol. (2020) 47:290-4. doi: 10.1111/1346-8138.15193
67. AbuHilal M, Walsh S, Shear N. Use of apremilast in combination with other therapies for treatment of chronic plaque psoriasis: a retrospective study. $J$ Cutan Med Surg. (2016) 20:313-6. doi: 10.1177/1203475416631328

68. Lee ES, Heller MM, Kamangar F, Park K, Liao W, Koo J. Hydroxyurea for the treatment of psoriasis including in HIV-infected individuals: a review. Psoriasis Forum. (2011) 17:180-7. doi: 10.1177/247553031117a00302

69. Lori F, Malykh A, Cara A, Sun D, Weinstein JN, Lisziewicz J, et al. Hydroxyurea as an inhibitor of human immunodeficiency virustype 1 replication. Science. (1994) 266:801-5. doi: 10.1126/science.79 73634

70. Salmon-Ehr V, Grosieux C, Potron G, Kalis B. Multiple actinic keratosis and skin tumors secondary to hydroxyurea treatment. Dermatol Basel Switz. (1998) 196:274.

71. Sauer GC. Combined methotrexate and hydroxyurea therapy for psoriasis. Arch Dermatol. (1973) 107:369-70. doi: 10.1001/archderm.10 7.3.369

Conflict of Interest: The authors declare that the research was conducted in the absence of any commercial or financial relationships that could be construed as a potential conflict of interest.

Publisher's Note: All claims expressed in this article are solely those of the authors and do not necessarily represent those of their affiliated organizations, or those of the publisher, the editors and the reviewers. Any product that may be evaluated in this article, or claim that may be made by its manufacturer, is not guaranteed or endorsed by the publisher.

Copyright (c) 2021 Arora, Das and Arora. This is an open-access article distributed under the terms of the Creative Commons Attribution License (CC BY). The use, distribution or reproduction in other forums is permitted, provided the original author(s) and the copyright owner(s) are credited and that the original publication in this journal is cited, in accordance with accepted academic practice. No use, distribution or reproduction is permitted which does not comply with these terms. 